\title{
TRANSFERENCIA DE TECNOLOGÍA EN LA INDUSTRIA MAQUILADORA: UN ENSAYO DE INTERPRETACIÓN Y BÚSQUEDA DE CONCEPTOS*
}

\author{
Por \\ Elena Salazar Sandoval**
}

\begin{abstract}
RESUMEN
A partir de los años ochenta se observan ciertos cambios en la industria maquiladora mexicana, como la segmentación de procesos productivos más complejos que implican la introducción de maquinaria y equipo sofisticado. Este cambio en la utilización de maquinaria de tecnología más avanzada es condición necesaria, aunque no suficiente, para abrir la perspectiva de la existencia de transferencia de tecnología (TT) en la maquiladora. El análisis de la TT se hace difícil de determinar, puesto que los trabajos que estudian el cambio tecnológico en la maquiladora manejan una gran variedad de conceptos con diferente significado y un mismo concepto de TT para una diversidad de nuevos aspectos. En este sentido, este trabajo presenta una propuesta de definición de conceptos que pretende ayudar a conocer el proceso de TT en la maquiladora, la etapa en que se encuentra, grado de asimilación y definición de conceptos. Con base en este planteamiento se aplicó una encuesta piloto en las maquiladoras de la industria eléctrica electrónica y metal-mecánica en la ciudad de Mexicali, que permite plantear supuestos sobre el proceso de Tr. Las conclusiones señalan que el cambio tecnológico en la maquila es de proceso y no de producto, que los conocimientos tecnológicos fundamentales están disponibles al personal que los maneja; se visualiza asimilación de tecnología, sin embargo, no se observa una etapa más avanzada de Tr como investigación y desarrollo.
\end{abstract}

\begin{abstract}
Since the 1980's the maquiladora industry in Mexico undergoes changes, such as a segmentation in productive processes that become more complex and involved the insertion of sophisticated machinery and equipment. This modification towards the use of leading technology is a necessary, but not sufficient condition for the existence of a technology transfer process in the maquiladora industry. Technology transfer is difficult to determine, because available studies that analize technology change in maquila use various concepts with different meanings. Technology transfer has been equaled with a wide variety of aspects of the new maquila. This paper presents an interpretative proposal and conceptual definitions that can help to understand technology transfer process, its stages, and the degree of assimilation. With
\end{abstract}

* Agradezco los valiosos comentarios que realizó a este trabajo el Dr. Raúl Femández, de la Universidad de California en Irvine.

** Investigadora del Instituto de Investigaciones Sociales. 
this approach a pilot inquiry was made in the electric, electronic and machinery and equipment metal products maquiladora industries in Mexicali.

The conclusions show that technology change in the maquila industry take place in the production processes, that technological knowledge is available to the operating personal, that some assimilation of technology is detectable, but a more advanced stage such as research and development of the technology is absent.

\section{INTRODUCCIÓN}

A partir de los años ochenta se observan ciertos cambios en la maquiladora mexicana, donde se ha pasado de procesos de simple ensamble manual a segmentación de procesos productivos más complejos, los cuales han implicado la introducción de maquinaria y equipo sofisticado que requieren de una mayor especialización en el trabajo y una nueva organización en los procesos productivos.

Este cambio en la utilización de maquinaria de tecnología más avanzada es condición necesaria, aunque no suficiente, para abrir la perspectiva de que existe transferencia de tecnología (TT) en la actual industria maquiladora.

El planteamiento anterior se refuerza con trabajos empíricos que señalan a nivel de indicadores globales la posible existencia de TT (Mertens y Palomares, 1988:170-198; Domínguez y Brown, 1989:215-223; González-Aréchiga y Ramírez, 1989:97-124). Sin embargo, no se ha realizado un estudio lo suficientemente pormenorizado que permita determinar si existe la TT en la maquila mexicana, su estructura y nivel de asimilación.

Así mismo, no se han implementado instrumentos de análisis que permitan conocer ¿qué se está transfiriendo?, ¿cómo se está transfiriendo? y Łquién lo está utilizando? Esto se hace difícil, puesto que en la literatura actual que estudia el cambio tecnológico en la maquiladora se maneja una gran variedad de conceptos con diferente significado y un mismo concepto de TT para definir una diversidad de nuevos aspectos que requieren ser uniformados. En este sentido, aquí se presenta una propuesta de interpretación y de definición de conceptos que pretende ayudar a entender el proceso de TT en la maquila, la etapa en que se encuentra, el grado de asimilación y los mecanismos por los cuales se transfiere.

\section{TRAYECTORIA DE LA INDUSTRIA MAQUILADORA EN MÉXICO}

La actual industria maquiladora en México no es la misma que hace veinte años. Para entender los cambios que ha experimentado hasta nuestros días, es preciso considerar la combinación y dinámica de factores económicos, tanto nacionales como internacionales. 
La maquiladora en México se inició en 1965 con el establecimiento del Programa de Industrialización Fronteriza (PIF), por medio del cual el gobierno mexicano buscó proporcionar empleo a la mano de obra desocupada, resultado de la cancelación del convenio de braceros. El funcionamiento del PIF permitió ensamblar y procesar componentes en México con materias primas y productos semielaborados de origen extranjero, exentos del pago de impuestos de importación y cuya producción era totalmente reimportada por el país de origen. Este programa cumplio en parte ${ }^{1}$ con el objetivo de crear empleo en la zona fronteriza, de tal manera que, en el periodo de 1966-1974, el número de empresas instaladas pasó de 12 a 455 y el personal ocupado de 3,107 a 75,975 respectivamente. ${ }^{2}$

De 1974 a 1976, la maquiladora se caracterizó por un lento crecimiento y desocupación de personal, provocado por el cierre de empresas o por el breve funcionamiento de algunas de ellas. En este periodo muchas maquiladoras regresaron a su país de origen con la misma facilidad con que se habían instalado, situación que las identificó como empresas inestables y fuertemente dependientes de la actividad económica internacional, especialmente con la de Estados Unidos.

En el periodo de 1977-1983 se inició un auge en la industria maquiladora que permitió resaltar las ventajas que trae consigo, tales como: la generación de empleos y divisas, un proceso de afianzamiento en el modelo de industrialización por exportaciones, así como la perspectiva de propiciar el desarrollo industrial fronterizo. No obstante, la maquiladora se siguio considerando como una industria transitoria. ${ }^{3}$

A partir de los affos ochenta se contemplan cambios importantes como:

a) El decreto del 15 de agosto de 1983 que plantea el fortalecimiento de la participación de un mayor número de secretarías de Estado y Juntas de Conciliación y Arbitraje como intermediarios en conflictos laborales y se porporcionan nuevos incentivos fiscales, de aranceles y aduaneros; además, se permite la venta de productos maquilados al interior del país y se crean escuelas oficiales para la capacitación de personal a nivel técnico medio.

b) La dinámica de la maquiladora en este periodo es relevante, ya que de 1983 a 1986 su participación en el total de la industria creció en un

1 Se dice que el objetivo se cumplió parcialmente, puesto que la maquiladora en esta etapa mayoritariamente emplé mano de obra femenina, esto es, que la ocupación nunca estuvo dirigida hacia la mano de obra masculina que fue la afectads al término del programa braceros y menos hacia los migrantes devueltos (ver Arriola Wog, 1980).

2 Datos del Instituto Nacional de Estadística Geografia e Informática, DNBOI.

3 Un trabajo más detallado sobre la evolución de la maquiladora se encuentra en Jorge Carrillo Viveros (1989:37-53). 
promedio de $11.7 \%$ con respecto al periodo de $1974-1982$, mientras que la industria nacional permaneció estancada. Así mismo, el empleo generado por la maquiladora aumentó $21.7 \%$ de $1982-1987 .^{4}$

c) En su estructura se percibe la incorporación del uso de maquinaria de tecnología avanzada; un cambio en los procesos de trabajo y en la composición ocupacional. En este periodola maquila ya se considera como una industria base en la frontera mexicana, y por primera vez, dentro de los lineamientos que debe observar se contempla el de elevar la capacitación de los trabajadores, impulsar el desarrollo industrial y la transferencia de tecnología. 5

Por otro lado, en el contexto internacional se advierte una reorganización mundial de la producción, cuya procedencia se basa en la necesidad de hacer frente a la crisis económica internacional y de encontrar un nuevo patrón más efectivo ante la competencia internacional entre las grandes potencias económicas mundiales, Japón, Estados Unidos, y los países de la Comunidad Económica Europea.

En la actualidad, las empresas compiten a nivel mundial para asegurar precio y calidad al mismo tiempo, y es aquí donde la tecnología juega un papel fundamental, dentro del cual los salarios bajos siguen siendo un factor muy importante para la existencia de estas empresas en países como México.

En este contexto de estrategias, la industria estadounidense pugna por recuperar su espacio competitivo en el mercado internacional, en el que contempla el traspaso de procesos productivos a los países anfitriones.

En lo que respecta al caso de México, concretamente por parte de las empresas estadounidenses, este cambio se contempla como un proceso meditado, donde el traslado de procesos productivos o equipos muy costosos, que requieren para su operación de un gasto en capacitación y entrenamiento para personal mexicano a nivel de ingenieros, gerentes o administrativos, se ve compensado por los bajos costos salariales.

Además, la productividad y calidad del trabajo del mexicano resulta ser tan eficiente, y en algunos casos mejor que la del estadounidense, puesto que bajo sistemas de organización de trabajo bien estructurados, el mexicano logra ser más creativo, puesto que está acostumbrado a trabajar con los elementos que tiene a la mano. Otro aspecto considerado por parte

4 Datos del INEGI.

5 La misma redacción del "Decreto para el fomento y operación de la industria maquiladora de exportación" es muy ambigua en lo que se refiere a la transferencia de tecnología. (Diario Oficial de la Federación, 22 de diciembre de 1989). 
de la industria estadounidense es el de ejercer un papel únicamente de supervisor en el funcionamiento de la empresa mexicana. ${ }^{6}$

En este contexto, donde por una parte la subcontratación internacional utiliza el capital transnacional para mantener su competitividad y, por la otra, el gobierno mexicano encuentra soluciones de corto plazo a la crisis económica, se está dando la reorientación de la maquiladora que se caracteriza por los siguientes aspectos: a) el uso de tecnologías avanzadas que básicamente se localizan en las industrias del sector eléctrico, electrónico y metal-mecánico; b) el proceso de adaptación de nuevas tecnologías en la industria electrónica es generalizado y heterogéneo, según del tipo de producto de que se trate; c) La automatización en los procesos productivos es parcial y selectiva, ya que se combinan procesos intensivos en capital y de mano de obra; d) La eficiencia en el trabajo es medida principalmente a través de la calidad y proliferan los círculos de calidad; e) los objetivos que se pretenden alcanzar con la introducción de nuevas tecnologías están vinculados con un mayor control en el proceso de producción para lograr alta calidad y regularidad en el trabajo, y disminuir costos no salariales en base a la reducción en mermas y el ahorro en inventarios, garantizar seguridad industrial y aprovechar la flexibilidad propia de estas tecnologías; f) la automatización de los procesos productivos no siempre se lleva a cabo con maquinaria de última producción, sino que básicamente se realiza por medio de reconversión de maquinaria vieja o el traslado de equipos computarizados desde fábricas de origen; g) un efecto cualitativo que se aprecia al introducir nuevas tecnologías es el cambio en la composición de la fuerza de trabajo, que se visualiza a favor de un mayor número de técnicos e ingenieros, así como un cambio en la demanda del trabajador a nivel obrero, en el cual se requiere de una mayor atención y limpieza en el proceso de trabajo y una mayor capacidad de pensamiento. (Mertens y Palomares, 1988; Domínguez y Brown, 1989).

\section{TRANSFERENCIA DE TECNOLOGÍA}

La discusión sobre el tema de la TT en la maquiladora resulta difícil porque, de hecho, los términos usados para identificarla hacen referencia a un amplio rango de diferentes conceptos e igualmente se identifica como TT a las nuevas facetas que actualmente está experimentando la maquiladora mexicana. En este sentido, es importante enfatizar algunos aspectos fundamentales:

6 Un trabajo que describe desde el punto de vista empresarial como está contemplado este traspaso de procesos productivos al caso de México, y que hace especial referencia a la TT, es el de Campbell, Richard y Jr. Hodak, Dennis P. (1988). 
Si bien es cierto que el uso de maquinaria con tecnología más avanzada es una condición necesaria para que se de un proceso de TT, este simple hecho no es suficiente. Es importante no confundir que un cambio tecno16 gico en la maquinaria utilizada forzosamente conlleva una mayor capacitación de los trabajadores, sin embargo, este hecho por sí solo no representa una adquisición de conocimientos tecnologicos.

En una reconversión industrial la mayor capacitación del obrero juega un papel muy importante, pero ésta, generalmente, sólo se limita a generar mayores niveles de conocimientos y responsabilidades dentro de los procesos productivos. Aquí no hay que olvidar que, por la misma estructura de los procesos de trabajo, el personal con posibilidades de acceso a los conocimientos tecnológicos básicamente es el personal técnico, es decir, aquel personal que está más en contacto con los conocimientos tecnológi$\cos$, que en este caso lo conforman los técnicos, ingenieros y gerentes, no los obreros, y es a este nivel de trabajadores donde se debe buscar la TT.

El traspaso de personal técnico de la maquiladora a industrias nacionales, por sí mismo, no es significartivo como TT, primero hay que investigar si este personal ha adquirido conocimientos tecnologicos dentro de la maquila. Así mismo, un caso a considerar como TT sería determinar qué personal con nivel técnico, una vez que ha adquirido conocimientos tecnologicos en la maquila, posteriormente forme su propia empresa 0 desarrolle procesos productivos en base a los conocimientos adquiridos.

Igualmente, en lo que se refiere a TT suelen confundirse los mecanismos a través de los cuales se transfiere la tecnología, con lo que debe considerarse como TT en sí. En este trabajo la TT se entiende como un proceso de adquisición de conocimientos tecnológicos por individuos y, a través de ellos por las organizaciones. El proceso de TT se considera consolidado $^{7}$ si de forma autónoma es capaz de reproducir, resolver problemas o generar innovaciones en procesos productivos o productos, a partir de los conocimientos adquiridos o desarrollados.

De acuerdo con la acepción anterior, si la TT es un proceso, en la trayectoria de su evolución se deben de ir dando las bases de la TT, la cuestión ahora es definir a partir de qué etapa se puede considerar que existe TT, aunque el proceso no esté totalmente consumado.

Para lograr una aproximación que permita conocer la TT en la maquila se plantea un proceso que comprende cinco etapas: tecnología disponible, asimilación, adaptación, investigación y desarrollo. Donde la quinta etapa

7 Se utiliza el término consolidado para definir de alguna manera un objetivo alcanzado, pero la Tr es un proceso continuo e inacabable, por lo tanto, solamente existe la perspectiva de la prolongación de la capacidad de conocimientos y mejoras en la tecnología. 
es la difusión. Las primeras cuatro son consecuentes, mientras que la etapa de difusión se considera parcialmente independiente, puesto que se puede dar sin seguir el orden de las anteriores. Así mismo, cada una de las etapas comprende mecanismos a través de los čuales se transfiere la tecnología (ver figura 1).

Para que se inicie la $\mathrm{TT}^{8}$ es condición necesaria que la tecnología esté disponible, esto es, que su uso no esté restringido sólo a determinado personal; además, se requiere que los conocimientos tecnológicos deben estar debidamente organizados, actualizados y en un lenguaje accesible al personal técnico.

Los mecanismos para que la tecnología quede disponible al receptor, básicamente están contenidos en documentación como manuales, especificaciones, disenfos, layouts, instrucciones de proceso, instrucciones de producto, procedimientos administrativos, etc.

Si el primer paso es la disponibilidad de la tecnología, para que la capacidad tecnológica se pueda desarrollar y consolidar se debe de partir de conocimientos y experiencias de terceros, puesto que la TT se debe tratar de identificar en los conocimientos de las personas y no estrictamente en otros indicadores (como menores costos y/o mayor productividad); po ello el desarrollo de la tecnología es inseparable de la formación de personal. En esta segunda etapa del proceso se plantea la asimilación, ya que la aparición de diversas formas de aprendizaje asociadas al proceso productivo llegan de forma gradual al receptor, que en este caso, la TT la proporciona una capacitación y adquisición de conocimientos tecnológicos en los técnicos, ingenieros y gerentes.

Así, la asimilación es entendida como el aprendizaje racional y sistemático por medio del cual se conoce la tecnología en su estado original, ya sea en el proceso de producción, equipos, condiciones de operación del producto de la planta, etc.

La asimilación tecnológica se puede dar a través de cuatromecanismos: a) capacitación, b) por cambios en el equipo, c) actualización, y d) aprendizaje en el trabajo (ver figura 1). La capacitación, se considera formal, cuando al receptor se le proporcionan conocimientos estrechamente relacionados con el funcionamiento de la planta, el producto o los procesos productivos. Esta capacitación debe formar parte del programa de la empresa y se puede dar dentro de la misma planta, en la empresa

8 De hecho la Tr se puede iniciar desde la transmisión de los conocimientos necesarios para el diseño y construcción de las plantas industriales; sin embargo, en este análisis no se está considerando, puesto que la mayoría de las plantas y naves industriales ya se encuentran construidas cuando la maquiladora inicia su funcionamiento en México. Esto indica que en la construcción de la planta, la empresa extranjera no tiene injerencia de forma directa, por lo tanto, no se contempla la transmisión de conocimientos tecnológicos. 


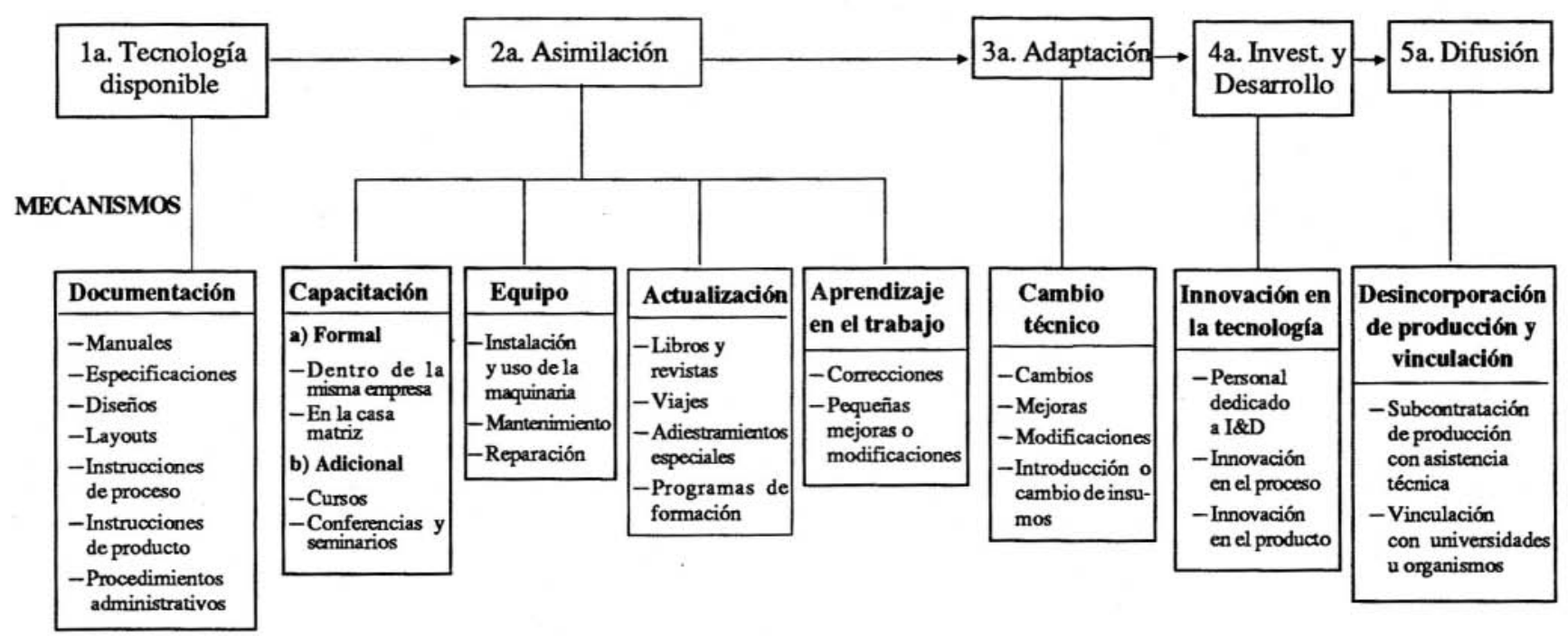

\section{FIGURA 1. Proceso de transferencia de tecnología.}


matriz en el extranjero o ser una combinación de ambas; mientras que la capacitación adicional proporciona al receptor un apoyo para desarrollar sus conocimientos, que puede ser mediante cursos especializados, asistencia a conferencias y seminarios, o bien, mediante cursos tan elementales como los de idiomas o computación, que le faciliten el acceso a los conocimientos tecnológicos.

La introducción de equipo de tecnología avanzada puede traer de manera intrínseca transferencia de Know-how, puesto que el receptor debe familiarizarse con el diseño y objetivos de la maquinaria. Pero el uso de este tipo de maquinaria debe de repercutir en la adquisición de conocimientos tecnológicos para que esté considerado como TT. Para ello, el receptor debe de entender los requerimientos de instalación, mantenimiento y reparación de la maquinaria y esto básicamente es adquirido mediante la capacitación y asistencia técnica, puesto que la documentación por sí sola proporciona poco conocimiento.

Una vez que la capacitación es adquirida por el receptor, ya sea de manera formal, adicional o por medio del equipo, ésta debe de mantenerse actualizada, a través de libros y revistas, viajes, adiestramiento especial o programas de formación de personal.

El cuarto mecanismo de asimilación de la tecnología se debe al aprendizaje en el trabajo, y es el flujo de conocimiento tecnológico derivado del desarrollo e incremento del adiestramiento, como resultado de la experiencia en la práctica de la producción en el trabajo, que puede darse sin que la capacitación sea condición necesaria. Esto se refleja en pequeñas mejoras, cambios o modificaciones.

La tercera etapa, que es la adaptación, permite la adecuación o modificación, parcial o total, de la tecnología transferida; es el conocimiento tecnológico producto de la capacitación y el aprendizaje en el trabajo, donde el factor habilidad humana es fundamental como contrapartida. Los mecanismos de la adaptación se reflejan en cambios, mejoras o modificaciones en aspectos como el proceso de producción, en el equipo, en el producto o en las condiciones de operación de la planta, etc., y en cambios en los insumos. Aquí es importante enfatizar que el término insumos se refiere a insumos productivos, es decir, materia prima para la producción; y que el cambio de un insumo, para que sea considerado como TT, debe ser producto del conocimiento tecnológico y no simplemente el resultado de la búsqueda de un sustituto en el mercado mexicano. Igualmente, el producto de esta adaptación debe repercutir en la extensión de estos conocimientos a la economía mexicana.

La investigación y desarrollo es la generación de algo nuevo, la introducción de un cambio tecnológico en el producto o en el proceso, mediante 
creación o mejora -innovación - fundamentado en los conocimientos científicos y técnicos adquiridos como resultado de las etapas anteriores. El mecanismo básicamente está dado por la asignación de personal a la investigación y desarrollo de la tecnología y se refleja en la innovación en el proceso o en el producto.

Por último, la difusión es la que permite desincorporar parte del proceso productivo, ésta se puede dar a través de la subcontratación a empresas nacionales, siempre y cuando implique proporcionar asistencia técnica por parte de la empresa que subcontrata, o por medio de la vinculación con universidades u organismos nacionales. El resultado de cualquiera de los dos mecanismos debe ser el mismo, adquisición de conocimientos tecnológicos para el receptor.

Este esquema de conceptos ${ }^{9}$ permite una aproximación para identificar la existencia de TT como proceso, su estructura, a través de qué mecanismos se está transfiriendo y en qué etapa se encuentra. Un segundo aspecto a determinar es el grado de asimilación, puesto que el desarrollo de la tecnología es inseparable de la formación de personal y de la aparición de diversas formas de aprendizaje que llegan de forma gradual al receptor. Es preciso intentar conocer el grado de aprendizaje adquirido, para ello se proponen cinco grados para medir la asimilación (ver figura 2):

- El primer grado es la dependencia completa, donde el personal que utiliza la tecnología desconoce el proceso de producción y especificaciones del producto. Las decisiones están totalmente en manos del propietario de la tecnología.

- Experiencia relativa. Hay experiencia en producir el producto, las decisiones locales se limitan a nivel de pregunta o sugerencia en base a criterios propios. No se conoce la flexibilidad del proceso.

- Creatividad incipiente. Se inician modificaciones y sustituciones mínimas en materias primas, diseños y especificaciones. Cualquier modificación requiere de la participación de la empresa poseedora de la tecnología.

- No dependencia. Se empieza a capitalizar el cambio menor, la mejora evolutiva, basándose en la operación misma de la planta.

- Finalmente, el último grado es la autosifuciencia, donde por extrapolación se generan productos y procesos nuevos (Giral, 1989).

9 Para la elaboración del esquema de conceptos y la figura 1, se consultaron los siguientes documentos: Enos y Park, 1988; Segal, 1987; Behrman y Wallender, 1987; Bell, 1985. 


\begin{tabular}{|l|l|l|l|l|}
\hline MECANISMOS & CAPACITACIÓN & EQUIPO & ACTUALIZACIÓN & $\begin{array}{c}\text { APRENDIZAJE } \\
\text { EN EL } \\
\text { TRABAJO }\end{array}$ \\
\hline $\begin{array}{l}\text { DEPENDENCIA } \\
\text { COMPLETA }\end{array}$ & & & & \\
\hline $\begin{array}{l}\text { DEPENDENCIA } \\
\text { RELATIVA }\end{array}$ & & & & \\
\hline $\begin{array}{l}\text { CREATIVIDAD } \\
\text { INCIPIENTE }\end{array}$ & & & & \\
\hline NO DEPENDENCIA & & & & \\
\hline AUTOSUFICIENCIA & & & & \\
\hline
\end{tabular}

FIGURA 2. Asimilación de tecnología. 


\section{TRANSFERENCIA DE TECNOLOGÍA Y ASIMILACIÓN TECNOLÓGICA EN LA INDUSTRIA ELÉCTRICA, ELECTRÓNICA Y METAL-MECÁNICA DE MEXICALI}

Dado el cambio que se observa en la maquiladora mexicana, surge interés por conocer si efectivamente se está dando TT y el grado de asimilación en que se encuentra. Para ello, en base al modelo antes expuesto, se realizó una encuesta piloto en 10 empresas escogidas al azar de las 40 maquiladoras que conforman el total de las ramas eléctrica, electrónica y metal-mecánica de Mexicali.

Los resultados son los siguientes:

- Mayoritariamente están constituidas por capital 100\% extranjero (7 de las diez empresas encuestadas).

- De los tres principales puestos a nivel técnico, el $78 \%$ cstá ocupado por mexicanos.

- Sólo una de las diez empresas tiene contrato de TT con su casa matriz. Éste involucra elementos tecnológicos de asistencia técnica y diseño de producto; mientras que las nueve restantes afirmaron que la asistencia técnica se las propociona la empresa de origen sin existir un contrato.

- Sólo una de las empresas encuestadas declaró no tener por escrito los manuales de procedimientos tecnológicos fundamentales.

- Con excepción de una empresa, todas tienen definido un programa de capacitación de recursos humanos en forma permanente. Los obreros son capacitados dentro de la misma maquiladora al igual que los supervisores, mientras que los ingenieros, administrativos y ejecutivos, además del entrenamiento dentro de la maquiladora, reciben capacitación en la empresa de origen y en otras instituciones dentro de la misma ciudad.

- En lo que se refiere a la procedencia de la información tecnológica, tres empresas afirmaron que la reciben por medio de revistas especializadas, cinco en ferias y exposiciones en el extranjero y dos por medio de las firmas de consultoría técnica en el extranjero. Las cuatro restantes reciben información tecnológica solamente a través de la empresa matriz.

- Todas reciben las materias primas de la empresa matriz.

- De las innovaciones tecnológicas que realiza la empresa matriz, seis de las empresas afirmaron que éstas son incorporadas en los procesos productivos correspondientes en la planta de México. 
- Durante los últimos tres años se realizaron modificaciones en los siguientes aspectos: todas al layout, nueve en los métodos de control de calidad, ocho en los materiales (Giral, 1989), siete en la maquinaria y cinco en el sistema de distribución. Dentro del concepto de "otras modificaciones", una afirmó haber realizado modificaciones en la estructura orgánica de la empresa, otra en el manejo de materiales y la última al diseño del producto.

- Sólo una de las empresas tiene personal asignado al diseño y evaluación de la estrategia tecnológica.

- Ninguna de las empresas proporciona asistencia técnica a proveedores externos.

\section{CONCLUSIONES}

Dado que se trata de una prueba piloto, resulta arriesgado inferir que los indicadores encontrados son comunes al conjunto de las maquiladoras de los sectores estudiados, pero a nivel indicativo se pueden concretar los siguientes supuestos:

- El cambio tecnológico en la maquiladora básicamente es de proceso y no de producto, puesto que los componentes esenciales de los insumos no sufren modificación, ya que estas decisiones se toman en la planta matriz. Se considera que el cambio tecnológico es de proceso, porque se presenta con un incipiente desarrollo tecnológico en sus componentes, como layout, ensamble, métodos de trabajo, etc., pero sin intervenir en el desarrollo de ingeniería de producto. Estos datos proporcionan el indicativo de que la TT debe ser igualmente de proceso y no de producto.

- Si casi todas las empresas disponen de la información fundamental sobre procedimientos tecnológicos, y el $78 \%$ del personal que maneja esta información es mexicano, es de suponer que la primera etapa de TT se está dando.

- En lo que respecta a la asimilación de tecnología, si casi todas las empresas cuentan con programas de capacitación de personal, dentro o fuera de la planta, además esta información se mantiene actualizada - por medio de revistas, ferias o exposiciones-, se puede afirmar que la asimilación de tecnología se está dando. Pero para conocer el grado de asimilación es necesario profundizar, investigando directamente al personal que es capacitado y que utiliza los conocimientos tecnológicos. 
- Si bien es cierto que la mayoría de las empresas han realizado modificaciones en casi todos, o en algunos, de los aspectos de los procesos productivos o en aspectos inherentes a la producción, esto no representa un indicativo de adaptación de la tecnología, puesto que la extensión de dichos cambios solamente es dentro de la misma maquila, es decir, que esta adaptación no se vincula con el ámbito nacional. Estas modificaciones más bien ratifican la asimilación de tecnología en un grado más avanzado, que representar una etapa posterior: la adaptación de la tecnología.

- Finalmente, el hecho de que ninguna de las empresas proporcione asistencia técnica a proveedores externos y sólo una tenga personal asignado al diseño y evaluación de la estrategia tecnológica, ratifica que las etapas de difusión e investigación y desarrollo de la tecnología no se están alcanzando.

\section{BIBLIOGRAFÍA}

ARRIOLA Wog, Mario. 1980. El programa mexicano de maquiladora. Instituto de Estudios, Universidad de Guadalajara.

BEHRMAN, Jack N. y Harvey W. Wallender. 1987. Transfers of Manufacturing Technology Within Multinational Enterprises. Ballenger Publish Company Cambridge. Massachusetts.

BELL, Martin. 1985. "Learning and the accumulation of industrial technological, capacity in developing countries". En: Fransman y King, Technological Capability in Developing Countries. Mc Millan.

CAMPBELL, Richard y Hodak, Dennis P. 1988. "Transferencia de tecnología para la industria maquiladora". In-Bond Industry, Banamex.

CARRILLO Viveros, Jorge. 1989. "Transformación en la industria maquiladora de exportación". En: Bernardo González-Aréchiga y Rocío Barajas (compiladores). Las maquiladoras: ajuste estructural y desarrollo regional. COLEF.

DOMÍNGUEZ, Lilia y Brown Flor. 1989. "Nuevas tecnologías en la industria maquiladora de exportación". Comercio Exterior. Vol. 39 , no. 3, marzo.

ENOS, J. LL y W. H. Park. 1988. The Adoption and Diffusion of Imported Technology: The Case of Korea. Croom Helm Ltd. Provident House, Australia. 
GIRAL, José. 1989. “Asimilación de Tecnología”. En: Marisol Pérez, Arturo Castaños y José Antonio Estera (compiladores). Articulacion tecnologica y productiva. México. Centro de Innovación Tecnológica de la UNAM.

GONZÁLEZ-ARÉCHIGA, Bernardo y José Carlos Ramírez. 1989. "Productividad sin distribución: cambio tecnológico en la maquiladora mexicana (1980-1986)". Frontera Norte 1, enero-junio. COLEF.

MERTENS, Leonard y Laura Palomares. 1988. "El surgimiento de un nuevo tipo de trabajador en la industria de alta tecnología. El caso de la electrónica". En: Esthela Gutiérrez (compiladora), Reestructuración productiva y clase obrera. México, Ed. Siglo XXI-UNAM.

SEGAL, Aaron. 1987. Learning by Doing Science and Technology in the Developing World. Westriev Press-Boulder and London. 